\title{
APPROXIMATION OF JACOBIAN INVERSE KINEMATICS ALGORITHMS
}

\author{
KRZYSZTOF TCHOŃ, JOANNA KARPIŃSKA，MARIUSZ JANIAK \\ Institute of Computer Engineering, Control and Robotics \\ Wrocław University of Technology, Janiszewskiego 11/17, 50-372 Wrocław, Poland \\ e-mail: \{krzysztof.tchon, joanna.karpinska, mariusz.janiak\}@pwr.wroc.pl
}

\begin{abstract}
This paper addresses the synthesis problem of Jacobian inverse kinematics algorithms for stationary manipulators and mobile robots. Special attention is paid to the design of extended Jacobian algorithms that approximate the Jacobian pseudoinverse algorithm. Two approaches to the approximation problem are developed: one relies on variational calculus, the other is differential geometric. Example designs of the extended Jacobian inverse kinematics algorithm for 3DOF manipulators as well as for the unicycle mobile robot illustrate the theoretical concepts.
\end{abstract}

Keywords: robot inverse kinematics, extended Jacobian, Jacobian pseudoinverse, approximation.

\section{Introduction}

This paper is devoted to the synthesis problem of inverse kinematics algorithms for redundant robotic manipulators and mobile robots. Our main objective is the design of an extended Jacobian algorithm approximating in a prescribed sense the Jacobian pseudoinverse algorithm. The origin of this problem comes from the now classical works (Roberts and Maciejewski, 1992; 1993). It is well known that the Jacobian pseudoinverse algorithm distinguishes by its performance and convergence; however, it lacks the property of repeatability (Klein and Huang, 1983). Let us recall that an inverse kinematics algorithm is referred to as repeatable if it transforms closed paths in the taskspace into closed paths in the configuration space: this implies that, if the robot works cyclically, then, at every repetition of an inverse kinematics problem in the cycle, the solution provided by the repeatable algorithm will be the same. A geometric repeatability condition of inverse kinematics algorithms for manipulators was provided in (Shamir and Yomdin, 1988) in terms of the integrability property of the distribution associated with the algorithm. A continuation of the geometric study on repeatability appears in (Roberts and Maciejewski, 1994). An extension of the repeatability condition to mobile robots is found in (Tchoń, 2002). In both cases of manipulators as well as mobile robots, the extended Jacobian inverse kinematics algorithm is repeatable by design. The idea of the extended Jacobian inverse as a method of redundancy resolution in redundant manipulators comes from (Baillieul, 1985). A novel formulation of this idea incorporating the optimization of a secondary performance criterion, appeared in (Klein et al., 1995). Extended Jacobian algorithms for mobile robots were developed in (Tchoń and Jakubiak, 2006).

As we have already mentioned, this paper addresses the approximation problem of the Jacobian pseudoinverse by the extended Jacobian inverse. As a point of departure we have taken a finite dimensional map of a configuration space into a taskspace representing the kinematics of a manipulator or of a nonholonomic mobile robot. In order to clarify the latter point, let us consider a mobile robot whose motion is subject to velocity constraints in the Pfaffian form. The kinematics of such a robot are described by a driftless control system with outputs

$$
\left.\dot{q}=G(q) u=\sum_{i=1}^{m} g_{(} q\right) u_{i}, \quad y=k(q),
$$

where $q \in \mathbb{R}^{n}, y \in \mathbb{R}^{r}$ refer, respectively, to generalized coordinates and taskspace coordinates of the robot, while $u \in \mathbb{R}^{m}$ denotes the control. Suppose that admissible control functions are defined over a time interval $[0, T]$ and belong to a function space $\mathcal{X}$. Then, the kinematics of the mobile robot can be identified with the end point map of the control system (1) (Chitour and Sussmann, 1998; Tchoń and Jakubiak, 2006),

$$
K_{q_{0}, T}: \mathcal{X} \rightarrow \mathbb{R}^{r}, \quad K_{q_{0}, T}(u(\cdot))=k\left(\varphi_{q_{0}, T}(u(\cdot))\right),
$$

where $\left.q(t)=\varphi_{q_{0}, t}(u(\cdot))\right)$ denotes the state trajectory of the system (1) initialized at $q_{0}$ and driven by $u(\cdot)$. The 
kinematics (2) are defined on an infinite dimensional function space. However, for computational purposes, we use a finite dimensional representation $\mathbb{R}^{p}$ of the control functions, e.g., by taking coefficients appearing in their $p$ dimensional truncated Fourier expansions. In this way, a finite dimensional representation

$$
k_{q_{0}, T}: \mathbb{R}^{p} \rightarrow \mathbb{R}^{r}
$$

of the mobile robot kinematics is obtained, which can be further processed in exactly the same way as the manipulator kinematics.

Having introduced the kinematics map, we define the inverse kinematics problem and derive Jacobian inverse kinematics algorithms relying on the continuation method (Chitour and Sussmann, 1998; Tchoń, 2007). As a particular case, we get the Jacobian pseudoinverse and extended Jacobian algorithms. The approximation problem will be dealt with in two formulations. The first formulation includes the classical Roberts and Maciejewski statement of the problem, and its alternative statement obtained by embedding the inverses into a larger space. The approximation problem is stated as an optimization problem in a function space and solved using the method of calculus of variations, resulting in a system of partial differential equations for the augmenting kinematics map. These partial differential equations are nonlinear in the case of the classical formulation, and linear elliptic for the alternative one.

The second formulation of the approximation problem consists in transforming the problem into that of the approximation of a codistribution annihilating the Jacobian transpose by an integrable codistribution. The procedure of determining the augmenting kinematics map exploits differential geometric tools (Sluis et al., 1996; Janiak and Tchon, 2008) and reduces the synthesis of the augmenting kinematics map to solving a system of partial differential equations by the method of characteristics. The extended Jacobian inverses obtained within the calculus of variations formulation have been applied to two robotic manipulators with three degrees of freedom, labeled as Manipulator 1 and Manipulator 2, and compared with the Jacobian pseudoinverse being their prototype. The extended Jacobian inverse devised within the differential geometric framework has been used to drive the unicycle-type mobile platform. The degree of redundancy of all these kinematics equals 1 . Using the differential geometric approach, in (Janiak and Tchoń, 2008), an extended Jacobian inverse was provided for Manipulator 1 and for the chained form system in dimension 3 . In both these examples the augmenting kinematics function was found analytically. The case of unicycle is much more difficult and can be treated only numerically.

The composition of this paper is the following: Section 2 introduces inverse kinematics algorithms. Section 3 develops the calculus of variations approach. The differential geometric approach is exposed in Section 4 A derivation of optimal extended Jacobian inverses for manipulators and the unicycle mobile robot is accomplished in Sections 5 and 6. Section 7 concludes the paper.

\section{Basic concepts}

We shall study either the kinematics of a stationary redundant manipulator or a finite dimensional representation of the mobile robot kinematics (3),

$$
k: \mathbb{R}^{p} \rightarrow \mathbb{R}^{r},
$$

with $p$ degrees of freedom and $r$-dimensional taskspace $(p>r)$. Let $J(x)=\partial k(x) / \partial x$ denote the manipulator's Jacobian. Given the kinematics (4) and a desirable point $y_{d}$ in the taskspace, the inverse kinematics problem amounts to determining a joint position $x_{d}$ such that $k\left(x_{d}\right)=y_{d}$. A numerical solution of the inverse kinematics problem is often delivered by a Jacobian inverse kinematics algorithm. The following continuation method argument presents a convenient way of deriving Jacobian algorithms. Given an initial configuration $x_{0} \in \mathbb{R}^{p}$, we define in the joint space a curve $x(\theta)$, $\theta \in R$, passing through $x_{0}$, such that the taskspace error $e(\theta)=k(x(\theta))-y_{d}$ along this curve decreases exponentially with a prescribed decay rate $\gamma>0$, so that

$$
\frac{\mathrm{d} e(\theta)}{\mathrm{d} \theta}=-\gamma e(\theta) \text {. }
$$

The differentiation of the error in the above formula yields the differential equation

$$
J(x(\theta)) \frac{\mathrm{d} x(\theta)}{\mathrm{d} \theta}=-\gamma\left(k(x(\theta))-y_{d}\right),
$$

sometimes referred to as the Ważewski-Davidenko equation. This equation can be made explicit in the derivative $\mathrm{d} x(\theta) / \mathrm{d} \theta$ by using a right inverse $J^{\#}(x)$ of the Jacobian, i.e., $J(x) J^{\#}(x)=I_{p-r}$. Given such an inverse, we get a dynamic system

$$
\frac{\mathrm{d} x(\theta)}{\mathrm{d} \theta}=-\gamma J^{\#}(x(\theta))\left(k(x(\theta))-y_{d}\right)
$$

whose trajectory approaches a solution of the inverse kinematics problem, $x_{d}=\lim _{\theta \rightarrow+\infty} x(\theta)$. In practice, Jacobian inverse kinematics algorithms employ two right inverses of the Jacobian: either the Jacobian pseudoinverse or the extended Jacobian inverse. It is well known that at regular joint positions of the manipulator the Jacobian pseudoinverse is defined as

$$
J^{P \#}(x)=J^{T}(x)\left(J(x) J^{T}(x)\right)^{-1} .
$$

An alternative to the Jacobian pseudoinverse is the extended Jacobian inverse, introduced in the following 
way. Given the kinematics (4), we choose an augmenting kinematics map

$$
h: \mathbb{R}^{p} \longrightarrow \mathbb{R}^{p-r}, \quad \tilde{y}=h(x) .
$$

The map (9) allows us to define the extended kinematics

$$
l=(k, h): \mathbb{R}^{p} \longrightarrow \mathbb{R}^{p}, \quad \bar{y}=l(x),
$$

and the extended Jacobian $\bar{J}(x)=\partial l(x) / \partial x$. Wherever invertible, the extended Jacobian gives rise to the extended Jacobian inverse

$$
J^{E \#}(x)=\left.\bar{J}^{-1}(x)\right|_{r \text { first columns }}
$$

By design, the extended Jacobian inverse is a right inverse of the Jacobian that is annihilated by the Jacobian of the augmenting kinematics map, i.e.,

$$
J(x) J^{E \#}(x)=I_{r} \text { and } \frac{\partial h(x)}{\partial x} J^{E \#}(x)=0 .
$$

It is well known that the Jacobian pseudoinverse inverse kinematics algorithm distinguishes by its convergence, whereas the extended Jacobian inverse kinematics algorithm has the property of repeatability. A classical design problem of inverse kinematics algorithms consists in designing an extended Jacobian inverse approximating in a prescribed sense the Jacobian pseudoinverse. The next two section will be devoted to diverse formulations and solution methods of this problem.

\section{Optimal functional approximation}

In this section we formulate the approximation problem of the Jacobian pseudoinverse by an extended Jacobian inverse in terms of the minimization of an approximation error functional depending on the augmenting kinematics map. Two different error functionals will be defined following, respectively, (Roberts and Maciejewski, 1992; 1993) and (Tchoń, 2008).

3.1. Error functional 1. Given the inverses $J^{P \#}(x)$ and $J^{E \#}(x)$, the approximation problem consists in providing an augmenting map (9) that minimizes the approximation error

$$
\mathcal{E}_{1}(h)=\int_{\mathcal{M}_{1}}\left\|J^{P \#}(x)-J^{E \#}(x)\right\|_{F}^{2} \mathrm{~d} x
$$

over a singularity-free region $\mathcal{M}_{1} \subset \mathbb{R}^{p}$ of the joint space. The norm $\|M\|_{F}=\sqrt{\operatorname{tr}\left(M M^{T}\right)}$ denotes the Frobenius matrix norm. In order to show an explicit dependence of the error on $h(x)$, we observe that there exists a matrix $W(x)$ such that

$$
J^{P \#}(x)-J^{E \#}(x)=K(x) W(x) .
$$

The matrix $K(x)$ is an $p \times(p-r)$ matrix with orthonormal columns $\left(K^{T}(x) K(x)=I_{p-r}\right)$ spanning the Jacobian kernel, $(J(x) K(x)=0)$.

Next, thanks to the fact that both $J^{P \#}(x)$ and $J^{E \#}(x)$ are right inverses of the Jacobian, we compute the matrix $W(x)$

$$
W(x)=\left(\frac{\partial h(x)}{\partial x} K(x)\right)^{-1} \frac{\partial h(x)}{\partial x} J^{P \#}(x),
$$

and, finally, obtain the following explicit error functional:

$$
\begin{aligned}
& \mathcal{E}_{1}(h) \\
& =\int_{\mathcal{M}_{1}} \operatorname{tr}\left(\left(\frac{\partial h(x)}{\partial x} K(x)\right)^{-1} \frac{\partial h(x)}{\partial x} J^{P \#}(x)\right. \\
& \left.\quad \times J^{P \# T}(x)\left(\frac{\partial h(x)}{\partial x}\right)^{T}\left(\frac{\partial h(x)}{\partial x} K(x)\right)^{-T}\right) \mathrm{d} x .
\end{aligned}
$$

The functional 14 is an integral of a Lagrangian $L(x, \partial h(x) / \partial x)$, so the corresponding Euler equations (Gelfand and Fomin, 1963)

$$
\operatorname{tr} \frac{\partial}{\partial x}\left(\frac{\partial L}{\partial \frac{\partial h_{i}}{\partial x}}\right)=0,
$$

for $i=1, \ldots, p-r$, yield a collection of nonlinear partial differential equations for components of the augmenting kinematics map. Due to the form of the Lagrangian, a general derivation of these equations seems intractable.

3.2. Error functional 2. An alternative definition of the approximation error is based on natural embedding of the Jacobian inverses $J^{E \#}(x)$ and $J^{P \#}(x)$ into a pair of $n \times n$ matrices, and then solving the approximation problem for the embeddings. Specifically, outside singularities, $J^{E \#}(x)$ is embedded in the inverse extended Jacobian

$$
E_{1}(x)=\left[\begin{array}{c}
J(x) \\
\frac{\partial h(x)}{\partial x}
\end{array}\right]^{-1}=\left[\begin{array}{ll}
J^{E \#}(x) & Q(x)
\end{array}\right],
$$

$Q(x)$ denoting an $p \times(p-r)$ complementary matrix. The embedding of $J^{P \#}(x)$ relies on the isomorphism theorem

$$
E_{2}(x)=\left[\begin{array}{c}
J(x) \\
K^{T}(x)
\end{array}\right]^{-1}=\left[\begin{array}{ll}
J^{P \#}(x) & K(x)
\end{array}\right]
$$

The approximation error involves the Frobenius norm of a multiplicative measure of the difference between these embeddings,

$$
\mathcal{E}_{2}(h)=\int_{\mathcal{M}_{2}}\left\|E_{1}^{-1}(x) E_{2}(x)-I_{p}\right\|_{F}^{2} m(x) \mathrm{d} x,
$$


where $m(x)=\sqrt{\operatorname{det} M(x)}$ denotes the manipulability function, and the integration should be done over a regular region $\mathcal{M}_{2} \subset \mathbb{R}^{p}$ with $m(x) \mathrm{d} x$ playing the role of the volume form. After suitable mathematical manipulations, we obtain the approximation error functional

$$
\begin{aligned}
\mathcal{E}_{2}(h)= & \int_{\mathcal{M}_{2}} \operatorname{tr}\left(\frac{\partial h(x)}{\partial x} P(x)\left(\frac{\partial h(x)}{\partial x}\right)^{T}\right. \\
& \left.-2 \frac{\partial h(x)}{\partial x} K(x)+I_{p-r}\right) m(x) \mathrm{d} x,
\end{aligned}
$$

where we set

$$
\begin{aligned}
P(x) & =J^{P \#}(x) J^{P \# T}(x)+K(x) K^{T}(x) \\
& =\left(J^{T}(x) J(x)+K(x) K^{T}(x)\right)^{-1} .
\end{aligned}
$$

As in the previous subsection, the Lagrangian appearing in (19) has the form $L(x, \partial h(x) / \partial x)$. However, this time the Euler equations (15) can easily be derived in the explicit form

$$
\begin{array}{r}
\operatorname{tr} \frac{\partial}{\partial x}\left(m(x) P(x) \frac{\partial h_{i}}{\partial x}\right)-\operatorname{tr} \frac{\partial}{\partial x}\left(m(x) K_{i}(x)\right) \\
=\sum_{r=1}^{p}\left(\sum_{k=1}^{p} m(x) P_{k r}(x) \frac{\partial^{2} h_{i}(x)}{\partial x_{k} \partial x_{r}}\right. \\
+\sum_{k=1}^{p} \frac{\partial\left(m(x) P_{k r}(x)\right)}{\partial x_{r}} \frac{\partial h_{i}(x)}{\partial x_{k}} \\
\left.-\frac{\partial\left(m(x) K_{r i}(x)\right)}{\partial x_{r}}\right)=0
\end{array}
$$

for $i=1, \ldots, p-r$. We conclude that the optimality conditions (20) are tantamount to a collection of linear, elliptic partial differential equations that should be satisfied by the components of the map $h(x)$.

\section{Approximation of codistributions}

A different approach to defining an extended Jacobian inverse that approximates the Jacobian pseudoinverse comes from differential geometry and relies on designing an integrable codistribution that agrees with a given codistribution in certain regions of the manipulator joint space (Janiak and Tchoń, 2008). For this purpose, we have adopted some results recently obtained in control theory, in the context of approximate feedback linearization (Sluis et al., 1996). In order to introduce this approach, we begin with the Jacobian pseudoinverse (8) and associate to it a codistribution

$$
\Omega_{P}=\operatorname{span}_{C^{\infty}\left(\mathbb{R}^{p}\right)}\left\{\omega_{1}(x), \ldots, \omega_{p-r}(x)\right\}
$$

spanned by differential one-forms that annihilate the Jacobian transpose, i.e., $\omega_{i}(x) J^{T}(x)=0$ for $i=1, \ldots, p-r$.
Our objective will be to define an extended Jacobian inverse, depending on the augmenting kinematics map $h(x)=\left(h_{1}(x), \ldots, h_{p-r}(x)\right)$, such that the codistribution

$$
\Omega_{E}=\operatorname{span}_{C \infty\left(\mathbb{R}^{p}\right)}\left\{\mathrm{d} h_{1}(x), \ldots, \mathrm{d} h_{p-r}(x)\right\},
$$

spanned by differentials of components of the augmenting map, coincide with the codistribution $\Omega_{P}$ after restriction to prespecified submanifolds and along prespecified directions in $\mathbb{R}^{n}$. For this purpose, we shall define in $\mathbb{R}^{p}$ a foliation with $p-r$-dimensional leaves $E_{\alpha}$, parametrized by $\alpha \in \mathbb{R}^{r}$, with a reference zero-leaf $E_{0}$. Associated with this foliation is a homotopy $\Phi_{t}: \mathbb{R}^{p} \rightarrow \mathbb{R}^{p}$, where $t \in[0,1]$, such that $\Phi_{1}=\operatorname{id}_{\mathbb{R}^{p}}, \Phi_{0}: \mathbb{R}^{n} \rightarrow E_{0}$, and a composition law holds $\Phi_{s} \circ \Phi_{t}=\Phi_{s t}$. The homotopy $\Phi_{t}(x)$ respects the structure of the foliation in the sense that, for any leaf $E_{\alpha}$, its image lies in another leaf, $\Phi_{t}\left(E_{\alpha}\right) \subset E_{\alpha^{\prime}}$.

Given the homotopy, we introduce a vector field

$$
X(x)=\left.\frac{\mathrm{d} \Phi_{t}(x)}{\mathrm{d} t}\right|_{t=1}
$$

that will establish a characteristic direction in $\mathbb{R}^{p}$. Furthermore, it is easily shown that the vector field (21) fulfills the identity

$$
t \frac{\mathrm{d} \Phi_{t}(x)}{\mathrm{d} t}=X\left(\Phi_{t}(x)\right)
$$

making the homotopy $\Phi_{t}(x)$ a flow of the time-dependent vector field $X(x) / t$. To proceed it will be convenient to choose in $\mathbb{R}^{p}$ specific coordinates $x=(y, z)$ such that $z \in$ $\mathbb{R}^{p-r}$ varies along a leaf, $y \in \mathbb{R}^{r}$ is constant on every leaf, and the reference leaf is characterized in coordinates by $(0, z)$. We shall assume that, on the leaf $E_{\alpha}$, the one-forms spanning $\Omega_{P}$ are given in coordinates $(y, z)$ as $\left.\omega_{i}\right|_{E_{\alpha}}=$ $d z_{i}$, while the generators of the codistribution $\Omega_{E}$ take the form

$$
\bar{\omega}_{i}=\sum_{j=1}^{p-r} B_{i j}(x) \mathrm{d} h_{j}(x),
$$

for $i=1, \ldots, p-r$.

After all these preparations, we are ready to make the following statement of the approximation problem of the Jacobian pseudoinverse by an extended Jacobian inverse: Design an augmenting kinematics map $h(x)=$ $\left(h_{1}(x), \ldots, h_{p-r}(x)\right)$ such that $h(0, z)=z$, the distributions $\Omega_{P}$ and $\Omega_{E}$ coincide after restriction to the leaves of the foliation $\left\{E_{\alpha}\right\}$

$$
\left.\bar{\omega}_{i}\right|_{E_{\alpha}}=\left.\omega_{i}\right|_{E_{\alpha}},
$$

and agree along the direction of the vector field $X(x)$,

$$
\omega_{i}(x) X(x)=\bar{\omega}_{i}(x) X(x),
$$


for every $i=1, \ldots, p-r$. Now, let us observe that the identity

$$
\begin{aligned}
\bar{\omega}_{i}(x)= & \sum_{j=1}^{p-r} B_{i j}(x) \mathrm{d} h_{j}(x) \\
= & \sum_{j=1}^{p-r} \sum_{k=1}^{r} B_{i j}(x) \frac{\partial h_{j}(x)}{\partial y_{k}} \mathrm{~d} y_{k} \\
& +\sum_{j=1}^{p-r} \sum_{l=1}^{p-r} B_{i j}(x) \frac{\partial h_{j}(x)}{\partial z_{l}} \mathrm{~d} z_{l}
\end{aligned}
$$

results in

$$
\left.\bar{\omega}_{i}\right|_{E_{\alpha}}=\sum_{j=1}^{p-r} \sum_{l=1}^{p-r} B_{i j}(x) \frac{\partial h_{j}(x)}{\partial z_{l}} \mathrm{~d} z_{l} .
$$

Consequently, the requirement 23 is equivalent to

$$
B(x) \frac{\partial h(x)}{\partial z}=I_{p-r} .
$$

Next, setting $x=\Phi_{s}(y, z)$ and $F_{i}(x)=\omega_{i}(x) X(x)$, and using (22), we derive from (24) the equality

$$
\begin{aligned}
& F_{i}\left(\Phi_{s}(y, z)\right) \\
& =s \sum_{j=1}^{p-r} B_{i j}\left(\Phi_{s}(y, z)\right) \mathrm{d} h_{j}\left(\Phi_{s}(y, z)\right) \frac{\mathrm{d} \Phi_{s}(y, z)}{\mathrm{d} s} \\
& =s \sum_{j=1}^{p-r} B_{i j}\left(\Phi_{s}(y, z)\right) \frac{\mathrm{d} h_{j}\left(\Phi_{s}(y, z)\right)}{\mathrm{d} s}
\end{aligned}
$$

and conclude that

$$
s B\left(\Phi_{s}(y, z)\right) \frac{\mathrm{d} h \circ \Phi_{s}(y, z)}{\mathrm{d} s}=F\left(\Phi_{s}(y, z)\right),
$$

where $F(x)=\left(F_{1}(x), \ldots, F_{p-r}(x)\right)$. Finally, we set $H(s, y, z)=h\left(\Phi_{s}(y, z)\right)$ and, after suitable mathematical manipulations involving (25) and (26), we arrive at a system of $p-r$ identical partial differential equations parameterized by the $y$-coordinates,

$$
\frac{\partial H_{i}(s, y, z)}{\partial s}-\sum_{j=1}^{p-r} \frac{F_{j}\left(\Phi_{s}(y, z)\right)}{s} \frac{\partial H_{i}(s, y, z)}{\partial z_{j}}=0
$$

where $i=1, \ldots, p-r$.

This system can be solved by the method of characteristics. Indeed, it is easily seen that along the solution $z(s)$ of the characteristic equation

$$
\frac{\mathrm{d} z(s)}{\mathrm{d} s}=-\frac{F\left(\Phi_{s}(y, z)\right)}{s}, \quad z(0)=z_{0},
$$

the map $H(s, y, z(s))=$ const. Based on this fact, using the properties of the homotopy map $\Phi_{t}(x)$ and the assumption that on the reference leaf $h(0, z)=z$, we get

$$
\begin{aligned}
H(1, y, z(1)) & =h\left(\Phi_{1}(y, z)\right)=h(y, z) \\
& =H(0, y, z(0))=h\left(\Phi_{0}(y, z(0))\right) \\
& =h\left(0, z_{0}\right)=z_{0},
\end{aligned}
$$

and therefore $h(y, z)=z_{0}$.

The last identity means that in order to determine the augmenting kinematics map $h(x)$ we need to solve the implicit equation

$$
z(1)=z=\varphi(1, y, h(y, z)),
$$

where $\varphi\left(s, y, z_{0}\right)$ denotes the flow of (28). This can be done by integrating backward in time the characteristic equation (28) and finally restoring the original variable $x$.

\section{Manipulators}

In this section, we shall compute the optimal augmenting kinematics functions for two example kinematics of robotic manipulators with the degree of redundancy 1 . The performance of the obtained extended Jacobian inverse kinematics algorithms will be examined by computer simulations, and compared with the Jacobian pseudoinverse algorithm. Manipulator 1 illustrates the case when the optimal augmenting function can be found analytically. Manipulator 2 shows an application to the problem of standard tools for numerical computations. Both these examples demonstrate that the alternative formulation of the approximation problem is much more tractable computationally than the classical one.

5.1. Manipulator 1. We shall begin with the kinematics of a 3DOF planar manipulator (Tchoń, 2008), shown in Fig. 11 The manipulator has joint variables $\left(x_{1}, x_{2}, x_{3}\right)$. Its tasks variables $\left(y_{1}, y_{2}\right)$ describe the Cartesian position of the car $W 2$ with respect to a coordinate frame fixed to the manipulator's base. The joint variables $x_{1}$ and $x_{3}$ are driven by the motors $M 1$ and $M 3$. The position of $W 2$ along the runner $P 2$ depends both on $x_{3}$ and on the revolution angle of the toothed wheel $z 2$, coupled with the revolution angle of the toothed wheel $z 1$ through a transmission gear whose gear ratio is adjusted by $x_{2}$ driven

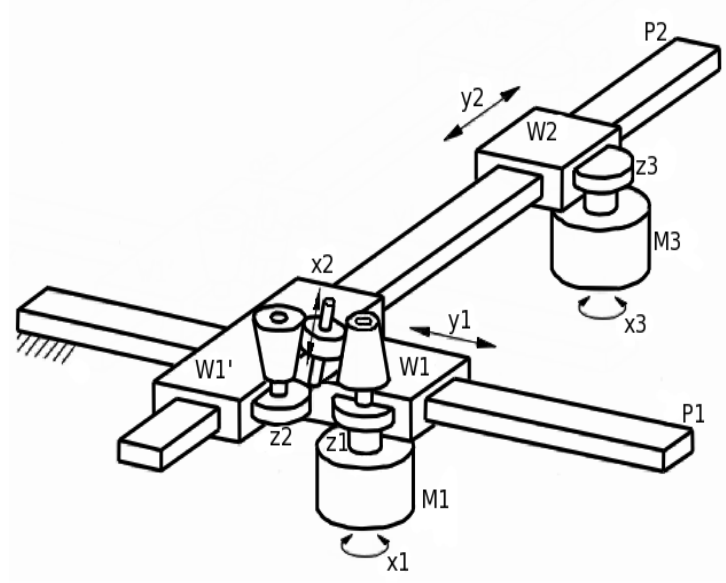

Fig. 1. Manipulator 1. 
by the motor $M 2$. In appropriate coordinates in the joint space of the manipulator, its kinematics are represented in the normal form

$$
k(x)=\left(x_{1}, x_{2}+x_{1} x_{3}\right) .
$$

A straightforward computation yields the Jacobian

$$
J(x)=\left[\begin{array}{ccc}
1 & 0 & 0 \\
x_{3} & 1 & x_{1}
\end{array}\right]
$$

the manipulability matrix

$$
M(x)=J(x) J^{T}(x)=\left[\begin{array}{cc}
1 & x_{3} \\
x_{3} & 1+x_{1}^{2}+x_{3}^{2}
\end{array}\right],
$$

the Jacobian kernel

$$
K(x)=\frac{1}{\sqrt{1+x_{1}^{2}}}\left(0,-x_{1}, 1\right)^{T},
$$

and, finally, the manipulability $m(x)=\sqrt{1+x_{1}^{2}}$.

5.1.1. Error functional 1. Our objective consists in defining an augmenting kinematics function $h(x)$ that minimizes the error functional (14). To simplify the computations, we shall look for this function in a specific form $h(x)=x_{3} f\left(x_{1}\right), f_{1}\left(x_{1}\right) \neq 0$. Then, the Euler equation corresponding to (14) reduces to the following second order ordinary differential equation involving $f\left(x_{1}\right)$ :

$$
\begin{aligned}
& 2 x_{3}\left(f^{2}\left(x_{1}\right)+4\left(1+x_{1}^{2}\right)\left(\frac{\mathrm{d} f\left(x_{1}\right)}{\mathrm{d} x_{1}}\right)^{2}\right. \\
& \left.-f\left(x_{1}\right)\left(5 x_{1} \frac{\mathrm{d} f\left(x_{1}\right)}{\mathrm{d} x_{1}}+\left(1+x_{1}^{2}\right) \frac{\mathrm{d}^{2} f\left(x_{1}\right)}{\mathrm{d} x_{1}^{2}}\right)\right)=0 .
\end{aligned}
$$

By means of the MATHEMATICA package, we have found an analytic solution

$$
f\left(x_{1}\right)=\frac{C_{1} \sqrt{1+x_{1}^{2}}}{\left(x_{1} \sqrt{1+x_{1}^{2}}+\sinh ^{-1}\left(x_{1}\right)+C_{2}\right)^{\frac{1}{3}}},
$$

$C_{1}$ and $C_{2}$ denoting some integration constants. Eventually, the optimal augmenting function is

$$
h(x)=x_{3} \frac{C_{1} \sqrt{1+x_{1}^{2}}}{\left(x_{1} \sqrt{1+x_{1}^{2}}+\sinh ^{-1}\left(x_{1}\right)+C_{2}\right)^{\frac{1}{3}}} .
$$

For a further comparison, we shall use the Taylor expansion of the denominator of (31) around $x_{1}=0$, retaining linear terms. After substituting $C_{1}=C_{2}=1$, the result is

$$
f\left(x_{1}\right) \cong\left(1-\frac{2}{3} x_{1}\right) \sqrt{1+x_{1}^{2}},
$$

yielding an approximate augmenting function

$$
h\left(x_{1}, x_{3}\right) \cong x_{3}\left(1-\frac{2}{3} x_{1}\right) \sqrt{1+x_{1}^{2}} .
$$

5.1.2. Error functional 2. Now we shall determine an augmenting kinematics function $h(x)$ that minimizes the error (19). We begin by computing the matrix

$$
P(x)=\left[\begin{array}{ccc}
1 & \frac{-x_{3}}{1+x_{1}^{2}} & \frac{-x_{1} x_{3}}{1+x_{1}^{2}} \\
\frac{-x_{3}}{1+x_{1}^{2}} & \frac{1+x_{1}^{2}+x_{3}^{2}+x_{1}^{4}}{\left(1+x_{1}^{2}\right)^{2}} & \frac{x_{1} x_{3}^{2}-x_{1}^{3}}{\left(1+x_{1}^{2}\right)^{2}} \\
\frac{-x_{1} x_{3}}{1+x_{1}^{2}} & \frac{x_{1} x_{3}^{2}-x_{1}^{3}}{\left(1+x_{1}^{2}\right)^{2}} & \frac{1+2 x_{1}^{2}+x_{1}^{2} x_{3}^{2}}{\left(1+x_{1}^{2}\right)^{2}}
\end{array}\right]
$$

The corresponding Euler equation (20) can be written as

$$
\begin{aligned}
& \sqrt{1+x_{1}^{2}} \frac{\partial^{2} h(x)}{\partial x_{1}^{2}}+\frac{1+x_{1}^{2}+x_{3}^{2}+x_{1}^{4}}{\left(1+x_{1}^{2}\right)^{3 / 2}} \frac{\partial^{2} h(x)}{\partial x_{2}^{2}} \\
& +\frac{1+2 x_{1}^{2}+x_{1}^{2} x_{3}^{2}}{\left(1+x_{1}^{2}\right)^{3 / 2}} \frac{\partial^{2} h(x)}{\partial x_{3}^{2}}-\frac{2 x_{3}}{\left(1+x_{1}^{2}\right)^{1 / 2}} \frac{\partial^{2} h(x)}{\partial x_{1} \partial x_{2}} \\
& -\frac{2 x_{1} x_{3}}{\left(1+x_{1}^{2}\right)^{1 / 2}} \frac{\partial^{2} h(x)}{\partial x_{1} \partial x_{3}}+\frac{2 x_{1}\left(x_{3}^{2}-x_{1}^{2}\right)}{\left(1+x_{1}^{2}\right)^{3 / 2}} \frac{\partial^{2} h(x)}{\partial x_{2} \partial x_{3}} \\
& +\frac{3 x_{1} x_{3}}{\left(1+x_{1}^{2}\right)^{3 / 2}} \frac{\partial h(x)}{\partial x_{2}}+\frac{2 x_{1}^{2} x_{3}-x_{3}}{\left(1+x_{1}^{2}\right)^{3 / 2}} \frac{\partial h(x)}{\partial x_{3}}=0 .
\end{aligned}
$$

We assume a boundary condition $h\left(x_{1}, x_{2}, 0\right)=0$ and, as before, set $h(x)=x_{3} f\left(x_{1}\right)$. Then the partial differential equation (34) reduces to a second order linear ordinary differential equation

$$
\frac{\partial^{2} h(x)}{\partial x_{1}^{2}}-\frac{2 x_{3}}{1+x_{1}^{2}} \frac{\partial h(x)}{\partial x_{1}}+\frac{2 x_{1}^{2}-1}{\left(1+x_{1}^{2}\right)^{2}} f\left(x_{1}\right)=0 .
$$

Again, with the help of the MATHEMATICA package, we obtain an analytic solution $f\left(x_{1}\right)=$ $\left(a x_{1}+b\right) \sqrt{1+x_{1}^{2}}$ of (35), where $a, b$ are integration constants. As a result, the optimal augmenting kinematics function takes the following form:

$$
h_{a, b}(x)=x_{3}\left(a x_{1}+b\right) \sqrt{1+x_{1}^{2}} .
$$

By a comparison of (33) and (36), we notice that in the case of Manipulator 1, despite minimizing different approximation errors, the resulting optimal augmenting kinematics function (36) can be regarded as an approximate of the function 32.

5.2. Manipulator 2. Our second example involves a 3DOF planar manipulator presented in Fig. 2, with kinematics

$$
k(x)=\left(x_{2}+l \cos x_{3} \quad x_{1}+l \sin x_{3}\right) .
$$

The manipulator's Jacobian, the manipulability matrix, the manipulability function and the Jacobian kernel are 




Fig. 2. Manipulator 2.

computed as follows:

$$
\begin{aligned}
& J(x)=\left[\begin{array}{ccc}
0 & 1 & -l \sin x_{3} \\
1 & 0 & l \cos x_{3}
\end{array}\right], \\
& M(x)=\left[\begin{array}{cc}
l+l^{2} \sin ^{2} x_{3} & -l^{2} \sin x_{3} \cos x_{3} \\
-l^{2} \sin x_{3} \cos x_{3} & l+l^{2} \cos ^{2} x_{3}
\end{array}\right], \\
& m(x)=\sqrt{1+l^{2}}, \\
& K(x)=\frac{1}{m(x)}\left(-l \cos x_{3}, \quad l \sin x_{3}, \quad 1\right)^{T} .
\end{aligned}
$$

Our task is to find an augmenting kinematics function $h(x)$ minimizing solely the error (18). After suitable substitutions, we arrive at the Euler equation (20),

$$
\operatorname{tr} \frac{\partial}{\partial x}\left(R(x) \frac{\partial h(x)}{\partial x}\right)=0,
$$

where $R(x)=m(x) P(x)$. The coefficients of this matrix, computed for the unit arm length $l=1$ of the manipulator, are listed below:

$$
R(x)=\frac{\sqrt{2}}{2}\left[\begin{array}{ccc}
\frac{7-\cos \left(2 x_{3}\right)}{4} & \frac{\cos x_{3} \sin x_{3}}{2} & -\frac{\cos x_{3}}{2} \\
\frac{\cos x_{3} \sin x_{3}}{2} & \frac{7+\cos \left(2 x_{3}\right)}{4} & \frac{\sin x_{3}}{2} \\
-\frac{\cos x_{3}}{2} & \frac{\sin x_{3}}{2} & \frac{3}{2}
\end{array}\right] .
$$

Due to computation difficulties, let us assume that $h(x)=h\left(x_{2}, x_{3}\right)$. Then $R(x)$ reduces to

$$
R(x)=\frac{\sqrt{2}}{2}\left[\begin{array}{cc}
\frac{7+\cos \left(2 x_{3}\right)}{4} & \frac{\sin x_{3}}{2} \\
\frac{\sin x_{3}}{2} & \frac{3}{2}
\end{array}\right] .
$$

The numerical solution of (38) provided by the MATLAB PDE toolbox is shown in Fig. 3 The numerical solution

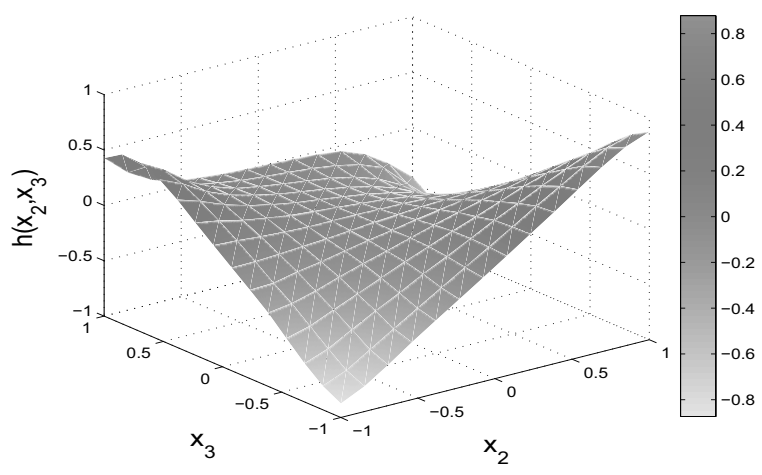

Fig. 3. Optimal augmenting function.

can be approximated by a third degree polynomial

$$
\begin{aligned}
& h\left(x_{2}, x_{3}\right) \\
& =0.0006 x^{3}-0.2205 y^{3}+0.3469 x^{2} y \\
& \quad+0.0009 x y^{2}+0.0005 x^{2}+0.0006 y^{2}-0.7501 x y \\
& \quad-0.0007 x+0.1381 y-0.0005
\end{aligned}
$$

presented in Fig. 4. Having obtained an analytic form of

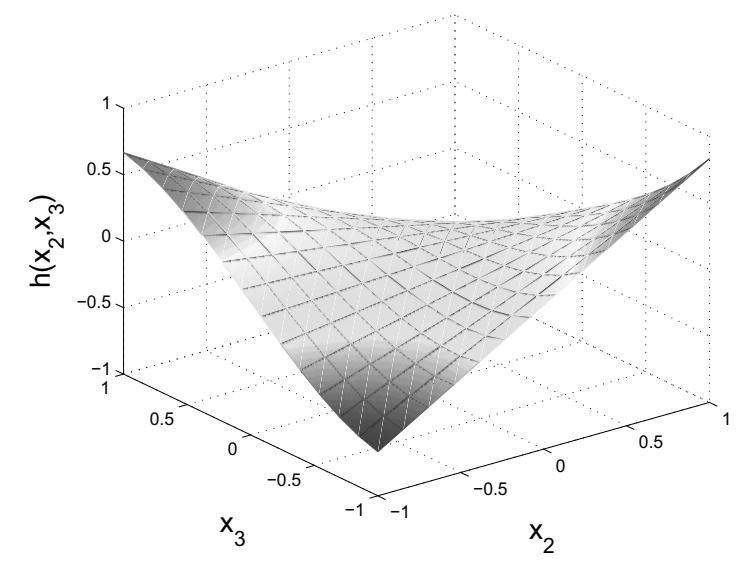

Fig. 4. Approximate augmenting function.

the augmenting function, we compute the extended Jacobian

$$
\bar{J}(x)=\left[\begin{array}{ccc}
0 & 1 & -\sin x_{3} \\
1 & 0 & \cos x_{3} \\
0 & j_{32}(x) & j_{33}(x)
\end{array}\right],
$$

where

$$
\begin{aligned}
j_{32}(x)= & 0.0012 x_{2}^{2}+0.6938 x_{2} x_{3}+0.0009 x_{3}^{2} \\
& +0.001 x_{2}-0.7501 x_{3}-0.0007, \\
j_{33}(x)= & -0.4410 x_{3}^{2}+0.3469 x_{2}^{2}+0.0018 x_{2} x_{3} \\
& +0.0012 x_{3}-0.0007 x_{2}+0.1381,
\end{aligned}
$$

and the corresponding extended Jacobian inverse. 
5.2.1. Computer simulations. In this subsection, we shall make a comparison of some performance aspects of the extended Jacobian inverse and the Jacobian pseudoinverse for Manipulator 2. The following simulation experiment focuses on the property of repeatability. To discover the repeatability, we have chosen four points in the taskspace: $A(1,1), B(1,2), C(0,2), D(0,1)$. The task of the inverse kinematics algorithm consists in driving the manipulator along a closed path starting at $A$ and returning to $A$ after passing through $B, C$, and $D$. At each step, the algorithm takes as an initial point the solution obtained at the previous step. The simulation results for $J^{E \#}(x)$ and $J^{P \#}(x)$ are collected in Table 1 . As can be seen, the extended Jacobian inverse kinematics algorithm based on the augmenting function (38) is repeatable, whereas the Jacobian pseudoinverse is not.

Now, we shall focus on a comparison of solutions of an inverse kinematics problem provided by the Jacobian pseudoinverse and extended Jacobian algorithms. In the testing problem, the desirable taskspace point of Manipulator 2 is $y_{d}=(0,2)$. As the initial condition we have chosen $x_{0}=(0,0, \pi / 2)$, which corresponds to the point $y=(0,1)$ in the taskspace.

In Figs. 5 and 6 the end effector paths are visualized, respectively, for the Jacobian pseudoinverse algorithm and the extended Jacobian algorithm. A comparison of the joint trajectories is presented in Figs. 7-9. The subscripts $\mathrm{P}$ and $\mathrm{E}$ refer, respectively, to the Jacobian pseudoinverse and to the extended Jacobian. As can be seen from these figures, both algorithms solve the inverse kinematic problem efficiently and generate very similar taskspace trajectories. However, it turns out that for larger distances between the initial and the desirable taskspace points the obtained joint trajectories may differ substantially.

Table 1. Simulation repeatability test.

\begin{tabular}{|lc|ccc|}
\hline \multicolumn{3}{|c|}{ Algorithm } & \multicolumn{3}{|c|}{ Extended Jacobian } \\
\hline A & $x_{0}$ & 0 & 0 & 1.5708 \\
B & $x_{1 f}$ & 0.1208 & 0.5235 & 1.0741 \\
C & $x_{2 f}$ & 1.1208 & 0.5235 & 1.0741 \\
D & $x_{3 f}$ & 1 & 0 & 1.5708 \\
A & $x_{4 f}$ & 0 & 0 & 1.5708 \\
\hline Algorithm & \multicolumn{3}{|c|}{ Jacobian } & pseudoinverse \\
\hline A & $x_{0}$ & 0 & 0 & 1.5708 \\
B & $x_{1 f}$ & 0.1132 & 0.5379 & 1.0904 \\
C & $x_{2 f}$ & 1.0432 & 0.7093 & 1.2759 \\
D & $x_{3 f}$ & 1.0198 & 0.1980 & 1.7701 \\
A & $x_{4 f}$ & 0.0529 & 0.3210 & 1.8976 \\
\hline
\end{tabular}

\section{Mobile robots}

We shall study the kinematics of the unicycle mobile platform shown in Fig. 10 A differential geometric extended Jacobian approximation of the Jacobian pseudoinverse will be derived and examined.

6.1. Unicycle. We let $q=\left(q_{1}, q_{2}, q_{3}\right)$ denote the position and orientation of the unicycle moving on a horizontal plane. The kinematics of the unicycle are represented by

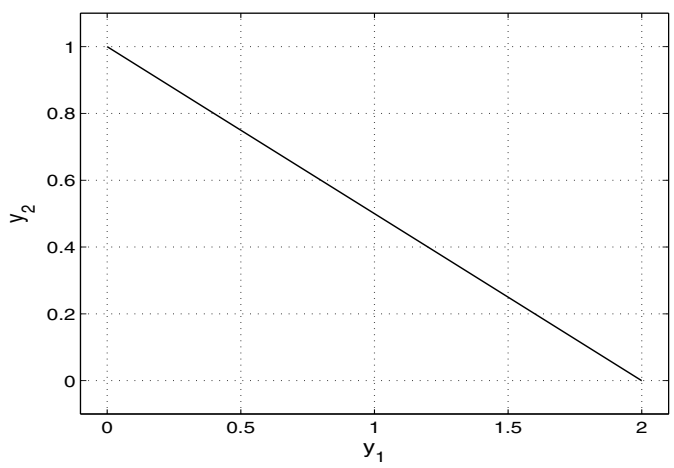

Fig. 5. Task space path of the Jacobian pseudoinverse.

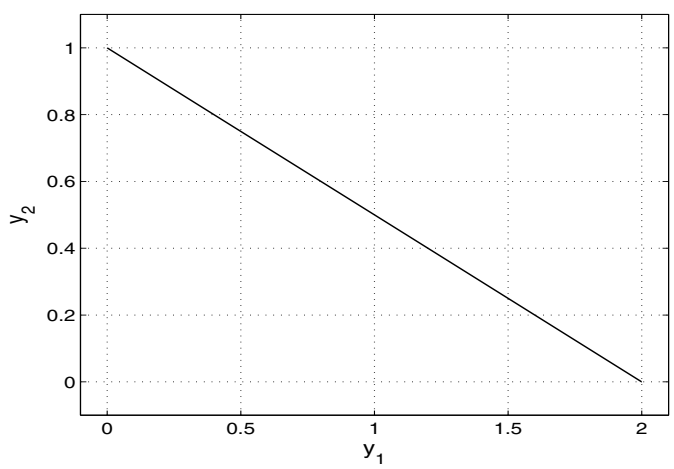

Fig. 6. Task space path of the extended Jacobian.

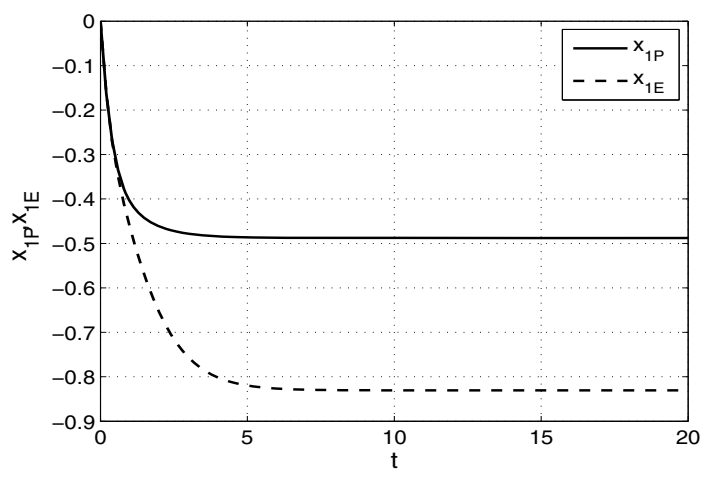

Fig. 7. $x_{1}(t)$ joint trajectories. 
the following control system with output:

$$
\dot{q}_{1}=u_{1} \cos q_{3}, \quad \dot{q}_{2}=u_{1} \sin q_{3}, \quad \dot{q}_{3}=u_{2}, \quad \mathrm{y}=q,
$$

where the controls $u_{1}$ and $u_{2}$ have the meaning of the longitudinal and angular velocities. Assuming the control horizon $T=2 \pi$, these controls will be chosen as

$$
u_{1}(t)=\lambda_{10}+\lambda_{11} \sin t, \quad u_{2}(t)=\lambda_{20}+\lambda_{21} \sin t
$$

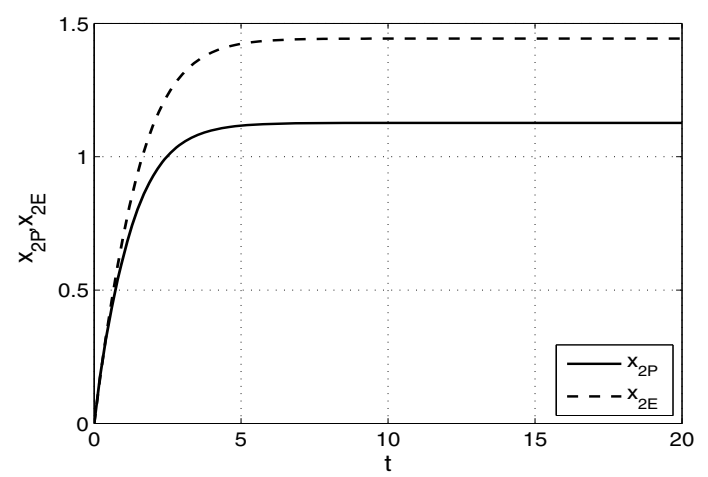

Fig. 8. $x_{2}(t)$ joint trajectories.

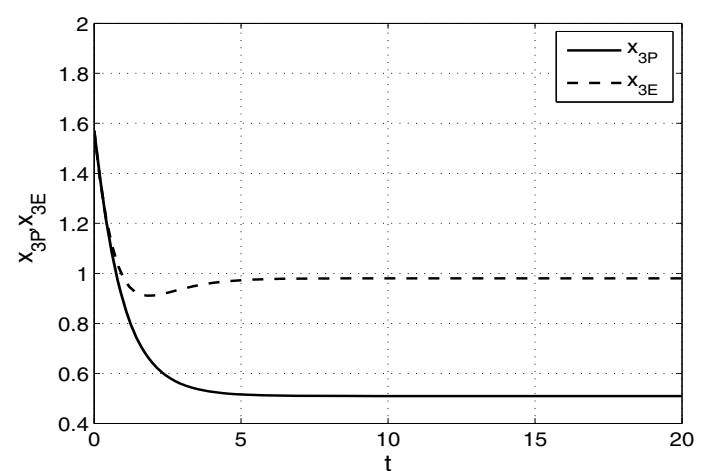

Fig. 9. $x_{3}(t)$ joint trajectories.

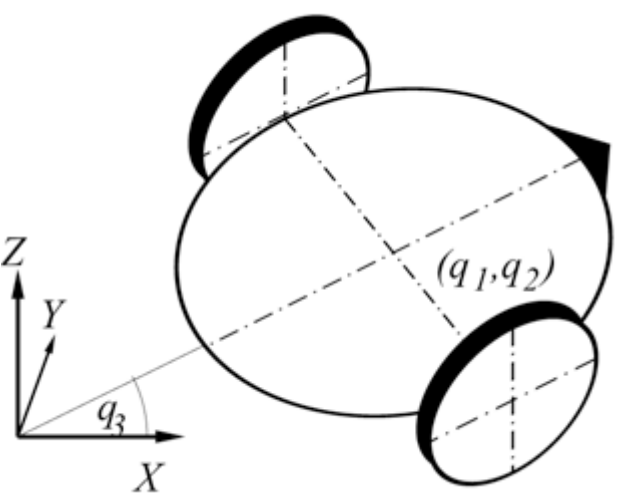

Fig. 10. Unicycle. so the control vector $\lambda=\left(\lambda_{10}, \lambda_{11}, \lambda_{20}, \lambda_{21}\right) \in \mathbb{R}^{4}$.

Suppose that initial coordinates of the unicycle $q_{0}=$ 0 . Then, the kinematics of the unicycle subject to the control (41) are the following:

$$
K_{q_{0}, T}(\lambda)=\left(q_{1 \lambda}(T), q_{2 \lambda}(T), q_{3 \lambda}(T)\right),
$$

where

$$
\begin{aligned}
q_{1 \lambda}(t) & =\int_{0}^{t}\left(\lambda_{10}+\lambda_{11} \sin s\right) \cos q_{3 \lambda}(s) \mathrm{d} s, \\
q_{2 \lambda}(t) & =\int_{0}^{t}\left(\lambda_{10}+\lambda_{11} \sin s\right) \sin q_{3 \lambda}(s) \mathrm{d} s, \\
q_{3 \lambda}(t) & =\int_{0}^{t}\left(\lambda_{20}+\lambda_{21} \sin s\right) \mathrm{d} s \\
& =\lambda_{20} t+\lambda_{21}(\cos t-1) .
\end{aligned}
$$

Given the kinematics (42), we compute the Jacobian

$$
J_{q_{0}, T}(\lambda)=\frac{\partial K_{q_{0}, T}(\lambda)}{\partial \lambda}=\left[J_{i j}(\lambda)\right]
$$

whose entries are

$$
\begin{aligned}
& J_{11}(\lambda)=\int_{0}^{T} \cos q_{3 \lambda}(s) \mathrm{d} s \\
& J_{12}(\lambda)=\int_{0}^{T} \sin s \cos q_{3 \lambda}(s) \mathrm{d} s \\
& J_{13}(\lambda)=-\int_{0}^{T}\left(\lambda_{10}+\lambda_{11} \sin s\right) \sin q_{3 \lambda}(s) s \mathrm{~d} s, \\
& J_{14}(\lambda)=-\int_{0}^{T}\left(\lambda_{10}+\lambda_{11} \sin s\right) \sin q_{3 \lambda}(s)(\cos s-1) \mathrm{d} s, \\
& J_{21}(\lambda)=\int_{0}^{T} \sin q_{3 \lambda}(s) \mathrm{d} s, \\
& J_{22}(\lambda)=\int_{0}^{T} \sin s \sin q_{3 \lambda}(s) \mathrm{d} s, \\
& J_{23}(\lambda)=\int_{0}^{T}\left(\lambda_{10}+\lambda_{11} \sin s\right) \cos q_{3 \lambda}(s) s \mathrm{~d} s, \\
& J_{24}(\lambda)=\int_{0}^{T}\left(\lambda_{10}+\lambda_{11} \sin s\right) \cos q_{3 \lambda}(s)(\cos s-1) \mathrm{d} s, \\
& J_{31}(\lambda)=0, \quad J_{32}(\lambda)=0, \quad J_{33}(\lambda)=1, \quad J_{34}(\lambda)=0 .
\end{aligned}
$$

The Jacobian (43) allows us to define the Jacobian pseudoinverse

$$
J_{q_{0}, T}^{P \#}(\lambda)=J_{q_{0}, T}^{T}(\lambda)\left(J_{q_{0}, T}(\lambda) J_{q_{0}, T}^{T}(\lambda)\right)^{-1} .
$$

Because $p-r=1$, the codistribution annihilating (44)

$$
\Omega_{P}=C^{\infty}\left(\mathbb{R}^{4}\right) \omega
$$

is spanned over smooth functions by the one-form $\omega(\lambda)=$ 
$\left(\omega_{1}(\lambda), \omega_{2}(\lambda), \omega_{3}(\lambda), \omega_{4}(\lambda)\right)$, whose components are

$$
\begin{aligned}
& \omega_{1}(\lambda)=\frac{J_{14}(\lambda) J_{22}(\lambda)-J_{12}(\lambda) J_{24}(\lambda)}{J_{11}(\lambda) J_{24}(\lambda)-J_{14}(\lambda) J_{21}(\lambda)}, \\
& \omega_{2}(\lambda)=1 \\
& \omega_{3}(\lambda)=0 \\
& \omega_{4}(\lambda)=\frac{J_{12}(\lambda) J_{21}(\lambda)-J_{11}(\lambda) J_{22}(\lambda)}{J_{11}(\lambda) J_{24}(\lambda)-J_{14}(\lambda) J_{21}(\lambda)} .
\end{aligned}
$$

It is easily checked that $\omega_{1}(\lambda)$ and $\omega_{4}(\lambda)$ are well defined provided that $\lambda_{10} \neq 0$. Now we define the foliation of the control space $\mathbb{R}^{4}$. Taking into account the well-definiteness of the codistribution $\Omega_{P}$, we choose the reference leaf $E_{a, 0}=a e_{1}+R e_{2}$, where $a \neq 0$ and $e_{i}$ denotes the $i$-th unit vector in $\mathbb{R}^{4}$, a general leaf having the form

$$
E_{a, \alpha}=E_{a, 0}+\alpha_{1} e_{1}+\alpha_{3} e_{3}+\alpha_{4} e_{4}
$$

for $\alpha_{1} \neq-a$. Following the procedure described in Section 4 we shall now introduce a homotopy map $\Phi_{t}: \mathbb{R}^{4} \rightarrow \mathbb{R}^{4}$, defined as

$$
\begin{aligned}
\Phi_{t}(\lambda) & =t \lambda+(1-t)\left(a e_{1}+\lambda_{11} e_{2}\right) \\
& =\left(t \lambda+(1-t) a, \lambda_{11}, t \lambda_{20}, t \lambda_{21}\right) .
\end{aligned}
$$

Next, we compute the characteristic vector field (21)

$$
X(\lambda)=\left.\frac{\Phi_{t}(\lambda)}{\mathrm{d} t}\right|_{t=1}=\left(\lambda_{10}-a, 0, \lambda_{20}, \lambda_{21}\right)
$$

and the function

$$
F(\lambda)=\omega(\lambda) X(\lambda)=\omega_{1}(\lambda)\left(\lambda_{10}-a\right)+\omega_{4}(\lambda) \lambda_{21} .
$$

After re-labeling the variables $\lambda$ in accordance with $y_{1}=\lambda_{10}-a, y_{2}=\lambda_{20}, y_{3}=\lambda_{21}, z=\lambda_{11}$, we obtain

$$
\Phi_{s}(y, z)=\left(s y_{1}+a, z, s y_{2}, s y_{3}\right)
$$

and derive the characteristic equation 28

$$
\begin{aligned}
\frac{\mathrm{d} z}{\mathrm{~d} s} & =-\frac{F\left(\Phi_{s}(y, z)\right)}{s} \\
& =\omega_{1}\left(\Phi_{s}(y, z)\right) y_{1}-\omega_{4}\left(\Phi_{s}(y, z) y_{3} .\right.
\end{aligned}
$$

By integrating this equation backward in time, we get the augmenting kinematics function $h(\lambda)=h(y, z)$. In conclusion, we compute the extended Jacobian

$$
\bar{J}_{q_{0}, T}(\lambda)=\left[\begin{array}{c}
J_{q_{0}, T}(\lambda) \\
\frac{\partial h(\lambda)}{\partial \lambda}
\end{array}\right],
$$

and the extended Jacobian inverse

$$
J_{q_{0}, 1}^{E \#}(\lambda)=\left.\bar{J}_{q_{0}, T}^{-1}(\lambda)\right|_{3 \text { first columns }} .
$$

6.2. Computer simulations. Computer simulations of the inverse kinematics algorithm (7) defined by (44) and (47) for the unicycle mobile platform have been run from two different starting positions and orientations $\mathrm{A}$ and $\mathrm{B}$ of the platform, characterized by the initial conditions $q_{0}^{A}=(5,0, \pi / 2)$ and $q_{0}^{B}=(-5,5, \pi / 2)$, with the time horizon $T=2 \pi$. In both cases, the initial control vector has been set to $\lambda_{0}=(0.5,-0.25,0.3,-0.25)$, the desirable taskspace point is $y_{d}=(1,0,-\pi / 2)$, and the algorithm decay rate has been fixed to $\gamma=0.1$. In the extended Jacobian inverse, the value $a=1$ of the parameter has been taken. Figures 11 and 13 demonstrate a solution of the inverse kinematic problem with the use of the Jacobian pseudoinverse algorithm (for the starting point A and B, respectively), and Figs. 12 and 14 accordingly, with the use of the extended Jacobian inverse. Paths of the unicycle platform in the $\left(q_{1}, q_{2}\right)$-plane are shown in the parts (a) of the figures, while convergence in the control space against the number of iterations in parts (b) and (c). Remarkably, the taskspace paths obtained from the point A are very similar, which cannot be observed for the point $B$.

\section{Conclusion}

This papers addressed the problem of optimal synthesis of inverse kinematics algorithms for robotic manipulators and mobile robots, focusing on devising an extended Jacobian algorithm by approximating the Jacobian pseudoinverse. Two approaches to the approximation problem were fostered: a calculus of variation based and a differential geometric. Within the former approach, we presented a traditional and an alternative formulation of the problem. Concerning the latter approach, we developed a synthesis procedure of the extended Jacobian algorithm, relying on the approximation of a codistribution by an integrable codistribution. Both approaches were illustrated with computer simulations.

\section{Acknowledgment}

This research has been supported by a statutory grant.

\section{References}

Baillieul, J. (1985). Kinematic programming alternatives for redundant manipulators, Proceedings of the 1985 IEEE International Conference on Robotics and Automation, St. Louis, LO, USA, pp. 722-728.

Chitour, Y. and and Sussmann, H. J. (1998). Motion planning using the continuation method, in J. Baillieul, S. S. Sastry and H. J. Sussmann (Eds), Essays on Mathematical Robotics, Springer-Verlag, New York, NY, pp. 91-125.

Gelfand, I. M. and Fomin, S. V. (1963). Calculus of Variations, Prentice-Hall, Englewood Cliffs, NJ. 




(a)

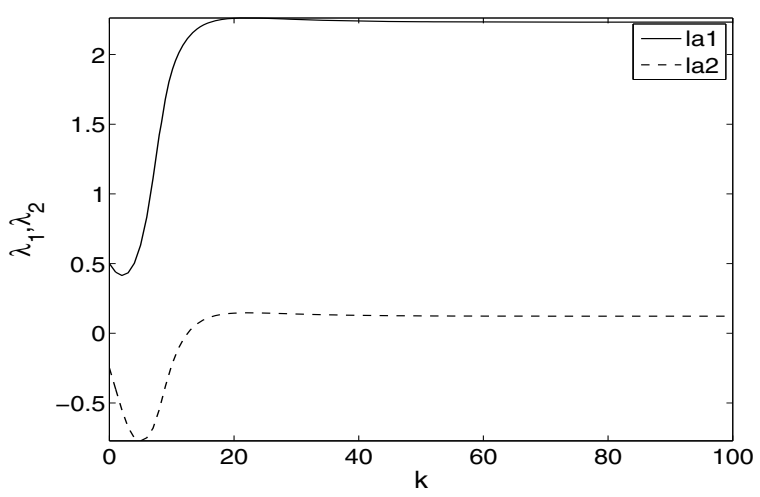

(b)

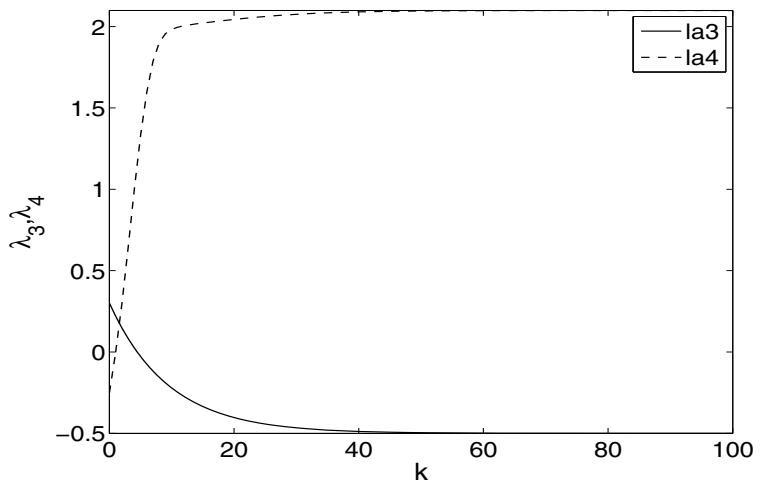

(c)

Fig. 11. Task space path and controls $\lambda$ with $J^{P \#}(\lambda)$ for the point A.

Janiak, M. and Tchoń, K. (2008). Extended Jacobian inverse kinematics and approximation of distributions, in J. Lenarcic and Ph. Wenger (Eds), Advances in Robot Kinematics, Springer Science+Business Media, Berlin, pp. 137-146.

Klein, Ch. A. and Huang, C. (1983). Review of the pseudoinverse control for use with kinematically redundant manipulators, IEEE Transactions on Systems, Man and Cybernetics 13(3): 245-250.

Klein, Ch. A., Chu-Jenq, C. and Ahmed, Sh. (1995). A new

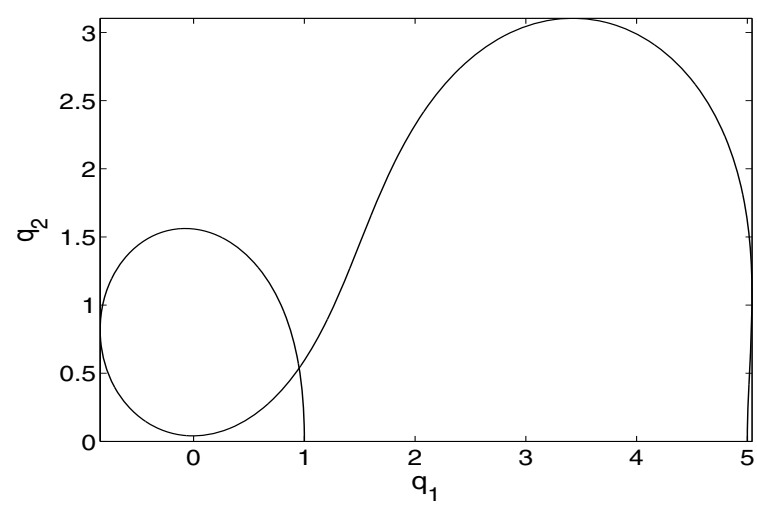

(a)

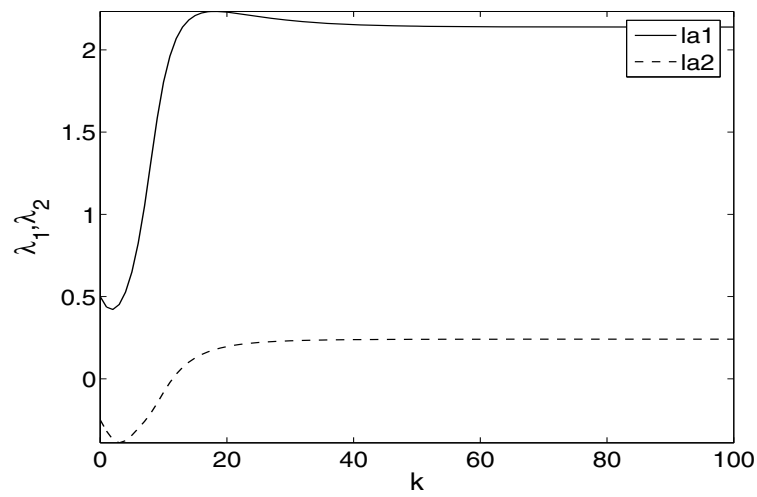

(b)

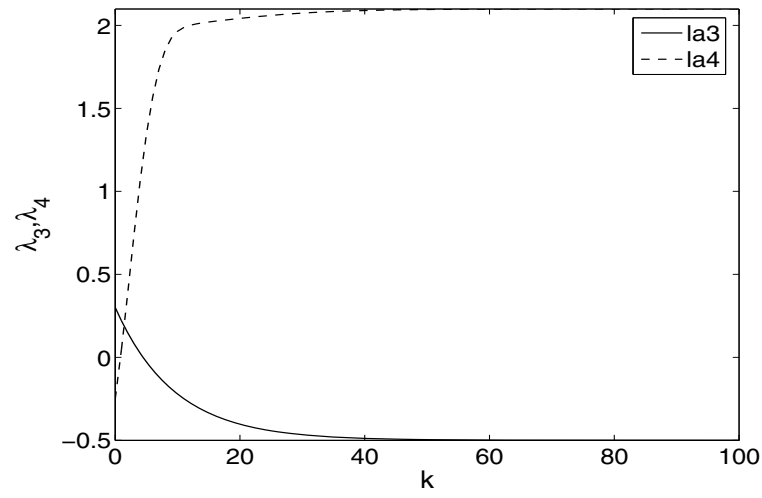

(c)

Fig. 12. Task space path and controls $\lambda$ with $J^{E \#}(\lambda)$ for the point A.

formulation of the extended Jacobian method and its use in mapping algorithmic singularities for kinematically redundant manipulators, IEEE Transactions on Robotics and Automation 11(1): 50-55.

Roberts, R. G. and Maciejewski, A. A. (1992). Nearest optimal repeatable control strategies for kinematically redundant manipulators, IEEE Transactions on Robotics and Automation 8(3): 327-337.

Roberts, R. G. and Maciejewski, A. A. (1993). Repeatable gene- 


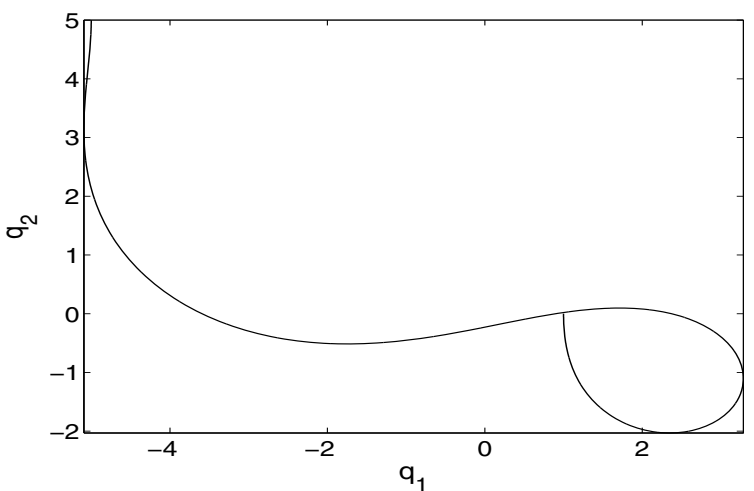

(a)

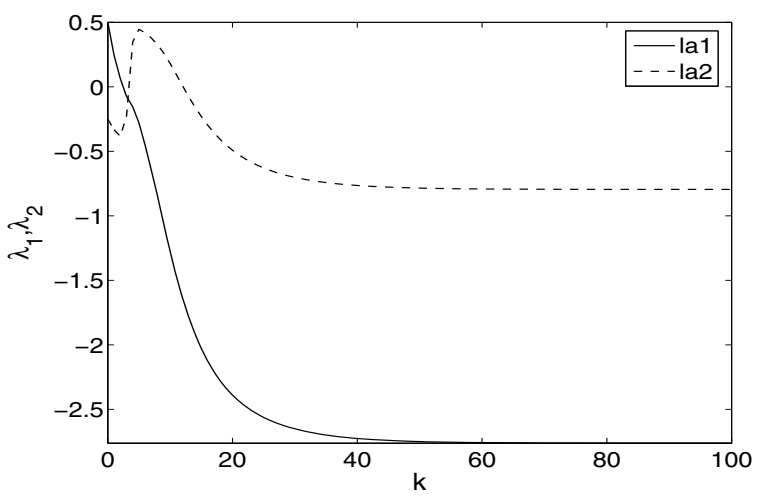

(b)



(c)

Fig. 13. Task space path and controls $\lambda$ with $J^{P \#}(\lambda)$ for the point B.

ralized inverse control strategies for kinematically redundant manipulators, IEEE Transactions on Automatic Control 38(5): 689-699.

Roberts, R. G. and Maciejewski, A. A. (1993). Singularities, stable surfaces, and the repeatable behavior of kinematically redundant manipulators, International Journal of Robotics Research 13(1): 207-213.

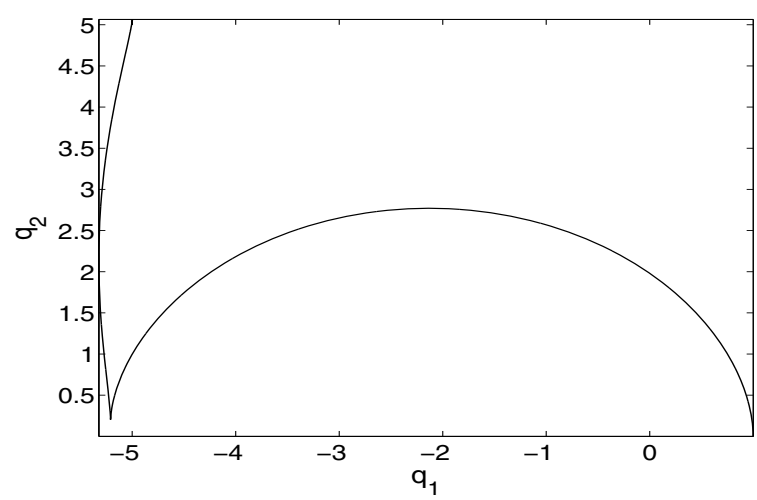

(a)



(b)

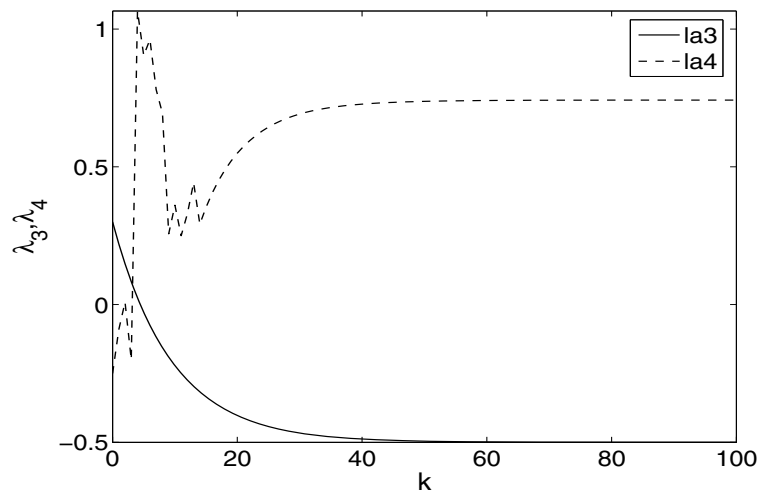

(c)

Fig. 14. Task space path and controls $\lambda$ with $J^{E \#}(\lambda)$ for the point B.

Shamir, T. and Yomdin, Y. (1988). Repeatability of redundant manipulators: Mathematical solution of the problem, IEEE Transactions on Automatic Control 33(11): 1004-1009.

Sluis, W. M., Banaszuk, A., Hauser, J. and Murray, R. M.(1996). A homotopy algorithm for approximating geometric distributions by integrable systems, Systems \& Control Letters 27(5): 285-291. 
Tchoń, K. (2002). Repeatability of inverse kinematics algorithms for mobile manipulators, IEEE Transactions on Automatic Control 47(8): 1376-1380.

Tchoń, K. (2007). Continuation method in robotics, Proceedings of the 7th Conference on Computer Methods and Systems, Cracow, Poland, pp. 17-24.

Tchoń, K. (2008). Optimal extended Jacobian inverse kinematics algorithms for robotic manipulators, IEEE Transactions on Robotics 28(6): 1440-1445.

Tchoń, K. and Jakubiak, J. (2006). Extended Jacobian inverse kinematics algorithm for non-holonomic mobile robots, International Journal of Control 79(8): 895-909.

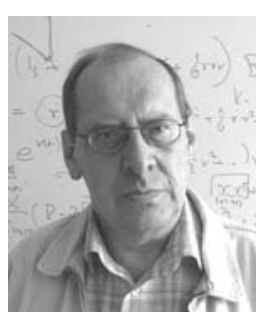

Krzysztof Tchoń received his Ph.D. and postdoctoral degrees from the Wrocław University of Technology, Poland, both in automation and robotics, respectively in 1976 and 1986. Currently he is a professor of automation and robotics and the head of the Unit of Fundamental Cybernetics and Robotics at the Institute of Computer Engineering, Control and Robotics, Wrocław University of Technology. Professor $\mathrm{K}$. Tchon is an author or coauthor of more than 150 publications. His research interests include geometric control theory as well as mathematical modeling and control of holonomic and nonholonomic robotic systems.

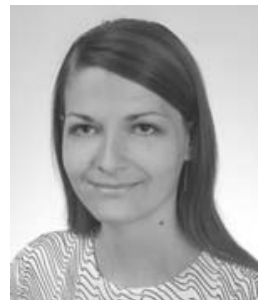

Joanna Karpińska received her M.Sc. degree in control engineering and robotics from the Wrocław University of Technology in 2007. Currently she is a Ph.D. student at the Institute of Computer Engineering, Control and Robotics of the same university. She is presently working on developing motion planning algorithms for holonomic and nonholonomic robotic systems.

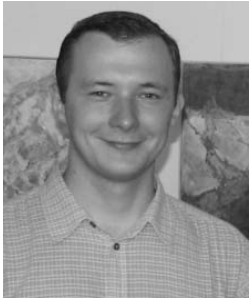

Mariusz Janiak received his M.Sc. degree in control engineering and robotics from the Wrocław University of Technology in 2005. Currently he is a Ph.D. student of the same university. His research interests include motion planning and control of mobile manipulators as well as social robots design. M. Janiak is an author of seven publications.

Received: 17 January 2009 Revised: 15 July 2009 